\title{
Cloreto de cálcio e radiação ultravioleta na conservação pós-colheita do tomate
}

\section{Calcium chloride and ultraviolet radiation in tomato postharvest conservation}

Cloruro de calcio y radiación ultravioleta en la conservación poscosecha del tomate

\section{Resumo}

O tomate (Lycopersicon esculentum) é um produto climatérico apresentando assim curto tempo de vida útil póscolheita. $\mathrm{O}$ uso exógeno de cloreto de cálcio $\left(\mathrm{CaCl}_{2}\right)$ e da radiação ultravioleta (UV), tem se mostrado eficiente na manutenção da firmeza e da qualidade de diversos frutos. Diante disso, este estudo objetivou avaliar os efeitos da aplicação exógena de cloreto de cálcio e radiação ultravioleta na conservação pós-colheita de frutos de tomate. Para isso, os frutos foram: submersos em cloreto de cálcio a $1 \%$ e expostos a radiação ultravioleta; imersos somente em cloreto de cálcio a $1 \%$; e frutos expostos apenas à radiação ultravioleta. Frutos não submersos no $\mathrm{CaCl}_{2}$ e não expostos a radiação UV foram utilizados como controle. Após aplicação dos tratamentos os frutos foram armazenados em condições ambientes e avaliados nos tempos 0,3, 6, 9, 12 e 15 dias após a colheita. Avaliou-se, perda de massa fresca, firmeza do fruto, cor da casca, teor de sólidos solúveis, $\mathrm{pH}$, acidez titulável total, razão entre os sólidos solúveis totais (SST) e acidez titulável (ATT). Verificou-se o aumento da perda de massa fresca, ao longo do tempo em todos os tratamentos. Frutos não imersos ou aqueles imersos em cloreto de $\mathrm{CaCl}_{2}+\mathrm{UV}$ apresentaram maior manutenção da firmeza. Pode-se concluir que, os frutos submetidos à radiação ultravioleta apresentaram menor perda de massa fresca ao final das análises, e o uso do cloreto de cálcio juntamente com a radiação UV mantiveram a firmeza dos frutos ao longo do armazenamento.

Palavras-chave: Armazenamento; Lycopersicon esculentum; Qualidade dos frutos; Radiação UV.

\section{Abstract}

Tomato (Lycopersicon esculentum) is a climacteric product with a short post-harvest shelf life. The exogenous use of calcium chloride $\left(\mathrm{CaCl}_{2}\right)$ and ultraviolet radiation (UV) has been shown to be efficient in maintaining the firmness and quality of several fruits. Therefore, this study aimed to evaluate the effects of exogenous application of calcium chloride and ultraviolet radiation on postharvest conservation of tomato fruits. For this, the fruits were: submerged in 
$1 \%$ calcium chloride and exposed to ultraviolet radiation; immersed in $1 \%$ calcium chloride only; and fruits exposed only to ultraviolet radiation. Fruits not submerged in $\mathrm{CaCl}_{2}$ and not exposed to UV radiation were used as controls. After application of treatments, fruits were stored under ambient conditions and evaluated at times 0, 3, 6, 9, 12 and 15 days after harvest. Fresh mass loss, fruit firmness, skin color, soluble solids content, $\mathrm{pH}$, total titratable acidity, ratio between total soluble solids (TSS) and titratable acidity (ATT) were evaluated. There was an increase in fresh mass loss over time in all treatments. Fruits not immersed or those immersed in $\mathrm{CaCl}_{2}+\mathrm{UV}$ chloride showed greater firmness maintenance. It can be concluded that the fruits subjected to ultraviolet radiation showed less loss of fresh mass at the end of the analysis, and the use of calcium chloride together with UV radiation maintained the firmness of the fruits throughout storage.

Keywords: Fruit quality; Storage; Lycopersicon esculentum; UV radiation.

\section{Resumen}

El tomate (Lycopersicon esculentum) es un producto climatérico con una corta vida útil poscosecha. Se ha demostrado que el uso exógeno de cloruro de calcio $\left(\mathrm{CaCl}_{2}\right)$ y radiación ultravioleta (UV) es eficaz para mantener la firmeza y la calidad de varias frutas. Por lo tanto, este estudio tuvo como objetivo evaluar los efectos de la aplicación exógena de cloruro de calcio y radiación ultravioleta en la conservación poscosecha de frutos de tomate. Para ello, los frutos fueron: sumergidos en cloruro cálcico al $1 \%$ y expuestos a radiación ultravioleta; sumergido en cloruro de calcio al $1 \%$ solamente; y frutas expuestas únicamente a la radiación ultravioleta. Se utilizaron como controles frutas no sumergidas en $\mathrm{CaCl}_{2}$ y no expuestas a radiación UV. Después de la aplicación de los tratamientos, los frutos se almacenaron en condiciones ambientales y se evaluaron en los tiempos 0, 3, 6, 9, 12 y 15 días después de la cosecha. Se evaluó la pérdida de masa fresca, la firmeza del fruto, el color de la piel, el contenido de sólidos solubles, el pH, la acidez titulable total, la relación entre los sólidos solubles totales (TSS) y la acidez titulable (ATT). Hubo un aumento en la pérdida de masa fresca con el tiempo en todos los tratamientos. Las frutas no sumergidas o las sumergidas en $\mathrm{CaCl}_{2}+$ cloruro UV mostraron mayor mantenimiento de firmeza. Se puede concluir que los frutos sometidos a radiación ultravioleta mostraron menor pérdida de masa fresca al final del análisis, y el uso de cloruro de calcio junto con radiación UV mantuvo la firmeza de los frutos durante todo el almacenamiento.

Palabras clave: Almacenamiento; Calidad de la fruta; Lycopersicon esculentum; Radiación UV.

\section{Introdução}

A produção de tomate (Lycopersicon esculentum) representa um dos maiores mercados agrícolas nacionais. O Brasil alcançou uma produção de 4 milhões de toneladas do fruto, sendo que a área plantada atingiu 56.874 hectares em (IBGE, 2021). Entretanto, assim como inúmeros produtos de origem vegetal, o cultivo do tomate é uma cultura de alto risco, visto que engloba perdas consideráveis desde o plantio até a pós-colheita.

O tomate é uma hortaliça altamente perecível após a colheita devido à grande fragilidade dos seus tecidos e ao aumento de sua atividade metabólica. Ao longo do período da pós-colheita do tomate ocorrem alterações na parede celular que causam amolecimento excessivo dos frutos. Esse amolecimento se deve ao afrouxamento da parede celular em conseqüência da degradação dos componentes da pectina e da hemicelulose. Nesse processo, ocorre aumento da pectina solúvel e diminuição da pectina insolúvel, ocasionando redução da firmeza (Song et al., 2016). Deste modo frutos de tomate demandam o emprego de tecnologias para a conservação da sua qualidade durante a pós-colheita.

Dentre as tecnologias que proporcionam a manutenção da qualidade pós-colheita de hortaliças, pode-se citar a utilização da radiação ultravioleta (UV) como uma das formas mais eficientes em reduzir essas perdas, a radiação UV encontra-se na faixa de 100 a $400 \mathrm{~nm}$, sendo que a radiação UV-C enquadra-se na ultravioleta curta, estabelecida entre 200 a $280 \mathrm{~nm}$ (Arenas, 2014).

O uso da radiação UV tem se propagado principalmente pela influência na redução da incidência e severidade de doenças e efeitos fisiológicos em tecidos vegetais. (Campos et al., 2015). Além disso, seu uso tem proporcionado o retardo de algumas mudanças associadas ao amadurecimento e as desordens fisiológicas. (Sanches et al., 2017).

Em estudos realizados por Moraes et., al (2021) com frutos de morango, a radiação UV mostrou-se promissora, ainda neste estudo, foi possível observar que frutos submetidos ao tratamento UV sofreram menos ataques de fungos e apresentaram um aumento de 9 dias de prateleira. O mesmo efeito foi observado por Almeida et., al (2019) onde a aplicação de UV durante 8 minutos revelou ser efetiva na manutenção da qualidade pós-colheita de caroços de pequi in natura. 
Outra técnica bastante empregada para a conservação do produto dá se por meio da aplicação de cloreto de cálcio $\left(\mathrm{CaCl}_{2}\right)$. O cloreto de cálcio quando aplicado na pós-colheita dos frutos atua, principalmente, na preservação da firmeza, na redução da taxa respiratória e produção de etileno, prolongando o período de vida útil do fruto (Yamamoto et al., 2011). A utilização da aplicação de $\mathrm{CaCl}_{2}$ mostrou-se eficiente em diversas culturas, como a exemplo do pimentão, tangerinas, maracujá, dentre outras (Paixão, et al. 2019; Vasconcelos et al., 2020; Bragança, 2021).

Diante do exposto, este trabalho tem como objetivo avaliar os efeitos da utilização de Cloreto de Cálcio $\left(\mathrm{CaCl}_{2}\right)$, da radiação ultravioleta (UV) e da interação entre eles em frutos de tomate durante o armazenamento.

\section{Metodologia}

Foi realizada uma pesquisa laboratorial (Estrela, 2018) onde foram utilizados frutos comerciais de tomate (Licopersicum esculentum), os quais foram adquiridos na central de abastecimento e horticultura na cidade de Aracaju, Sergipe (CEASA-SE). Os frutos foram lavados em água corrente e sanitizados com hipoclorito de sódio ( $300 \mathrm{ml} / \mathrm{L})$ por dez minutos e enxaguados em água corrente por 1 minuto. Posteriormente, os frutos foram mantidos em bancadas higienizadas, onde foram secos com papel toalha e permaneceram até a implantação do experimento.

O delineamento experimental foi inteiramente casualizado em esquema fatorial 4 × 6, com 3 repetições, totalizando 72 frutos. Foram avaliados 4 tratamentos: Controle ( $\left(\mathrm{em} \mathrm{CaCl}_{2} \mathrm{e} \mathrm{UV}\right.$ ); imersão em $\mathrm{CaCl}_{2}$ a $1 \%$ por 10 minutos $\left(\mathrm{CaCl}_{2}\right)$, exposição à radiação UV (lâmpada tubular NSK com $32 \mathrm{~W}$ de potência de saída e de 100-280nm de comprimento de onda) por 30 minutos (UV) e imersão em $\mathrm{CaCl}_{2}$ a $1 \%$ por 10 minutos mais exposição à radiação $\mathrm{UV}$ por 30 min $\left(\mathrm{CaCl}_{2}+\mathrm{UV}\right)$. Os frutos foram armazenados em condições ambientes $\left(25^{\circ} \mathrm{C}\right.$ e $80 \%$ UR) foram avaliados nos tempos $0,3,6,9,12$ e 15 dias após a colheita.

Em cada tempo de avaliação foram retirados 3 frutos de cada tratamento para avaliação da perda de massa fresca (PMF), firmeza, cor da casca, pH, teor de sólidos solúveis totais (SST), acidez total titulável (ATT) e relação SST/ATT.

A perda de massa fresca (PMF) dos frutos foi obtida a partir da pesagem destes desde a colheita até o último dia de armazenamento. Os frutos foram pesados em balança semianalítica modelo (modelo BG 8000 Max; GEHAKA) sendo os resultados expressos em gramas de massa fresca perdida.

Para a determinação da firmeza (F) utilizou-se o penetrômetro digital (modelo TR; Turoni), com ponteira de $3 \mathrm{~mm}$ de diâmetro. Foram realizadas três leituras em cada fruto inteiro, na região mediana e em lados opostos do mesmo. Os resultados obtidos foram expressos em Newton $(\mathrm{N})$.

Para o pH, extraiu-se $10 \mathrm{ml}$ de polpa de tomate, que foi acondicionada em Becker e posteriormente realizada a leitura com o auxílio de um peagâmetro digital.

A análise de sólidos solúveis (SST) foi realizada por meio de leitura utilizando-se refratômetro de banca digital (modelo RTD-45, Instrutherm), sendo os resultados expressos em graus ( ${ }^{\circ}$ Brix), de acordo com os procedimentos descritos por El-Bulk et al. (1997).

A ATT da polpa (\% ácido cítrico) foi determinada conforme a metodologia proposta pela AOAC (1992), utilizando o método titulométrico com $\mathrm{NaOH} 0,01 \mathrm{~N}$ e indicador de fenolftaleína, em que a titulação foi encerrada quando o pH atingiu 8,1.

A cor da casca (CC) foi determinada por reflectometria, utilizando-se de um colorímetro, (modelo CR-400; Konica Minolta), calibrado em superfície de porcelana branca sob condições de iluminação e expressa no módulo L., a*. e *b. A coordenada L refere-se à luminosidade dos frutos, *a refere-se a coordenada verde-vermelha (valores positivos para cores avermelhadas e negativos para cores esverdeada) e $* b$ refere-se a coordenada azul-amarela (valores positivos para tons amarelados e negativos para tons azulados) (Galindo et al., 2015). Foram realizadas três leituras por fruto em lados opostos da sua região equatorial. 
Os dados relacionados a firmeza e a perda de massa foram analisados por meio de análise de regressão. Os demais parâmetros foram submetidos à análise da variância $(\mathrm{p} \leq 0,05)$ e comparação de médias pelo teste de Tukey $(\mathrm{p} \leq 0,05)$, através do programa estatístico RBio.

\section{Resultados e Discussão}

A perda de massa aumentou significativamente ao longo do tempo para todos os tratamentos adequando-se a equação linear (Figura 1). Verificou-se perda de massa mais acentuada a partir do $6^{\circ}$ e $9^{\circ}$ dia de armazenamento, onde os frutos tratados com $\mathrm{CaCl}_{2}+\mathrm{UV}$ obtiveram maior perda, mantendo essa tendência no $12^{\circ}$ e $15^{\circ}$ dias de avaliação. Em um estudo desenvolvido por Paixão et al., (2020) foram encontrados resultados semelhantes para frutos de pimentão tratados com cloreto de cálcio, onde constatou-se um aumento de perda de massa dos frutos durante os primeiros seis dias de armazenamento, e perdas mais expressivas no nono dia. A pós-colheita do tomate é marcada pela intensificação dos processos fisiológicos como o aumento na taxa de transpiração, causando deste modo a desidratação e a consequente perda de massa dos frutos (Neto et al., 2016). A redução de água nos frutos, que ocorre devido à transpiração do fruto, resulta em amolecimento durante o armazenamento (Chien et al., 2013; Khaliq et al., 2015) dessa foram a perda de massa dos tomates observada no presente estudo pode ter sido resultante da transpiração dos mesmos durante o periodo de armazenamento. A transpiração em alta intensidade também é um dos fatores que podem interferir diretamente na qualidade dos frutos, provocando alterações na aparência como, por exemplo, o murchamento, ocasionando assim a diminuição da aceitabilidade do produto (Chien et al., 2013; Khaliq et al., 2015).

Ao longo do tempo de armazenamento verificou-se redução da firmeza dos frutos de tomates nos diferentes tratamentos (Figura 3). A perda de firmeza observada com o passar do tempo de armazenamento pode estar relacionada à ação das enzimas pectina metil esterase e poligaracturonase, as quais degradam a parede celular, principalmente as pectina e que são responsáveis pela estruturação e manutenção da firmeza dos frutos em todo seu ciclo de vida (Chitarra \& Chitarra, 2005). Frutos do tratamento $\mathrm{CaCl}_{2}$ e os que foram imersos em $\mathrm{CaCl}_{2}$ e expostos à radiação UV apresentaram maior firmeza após os 15 dias de armazenamento (Figura 3). Essa manutenção da firmeza esta relacionada à função do cálcio em formar pectato de cálcio, isso se dá devido à incorporação deste mineral à estrutura da parede celular, contribuindo dessa forma para a manutenção da qualidade pós-colheita de frutos. (Linhares et al., 2007). Os benefícios do uso de sais de cálcio na manutenção da qualidade de frutos foram estudados também por Carnelossi et al. (2021) onde foi observado o efeito positivo do lactato de cálcio a $6 \%$ na manutenção da firmeza de mamão minimamente processado durante o armazenamento. 
Figura 1: Perda de massa de frutos de tomate durante o armazenamento e submetidos a diferentes tratamentos pós-colheita.
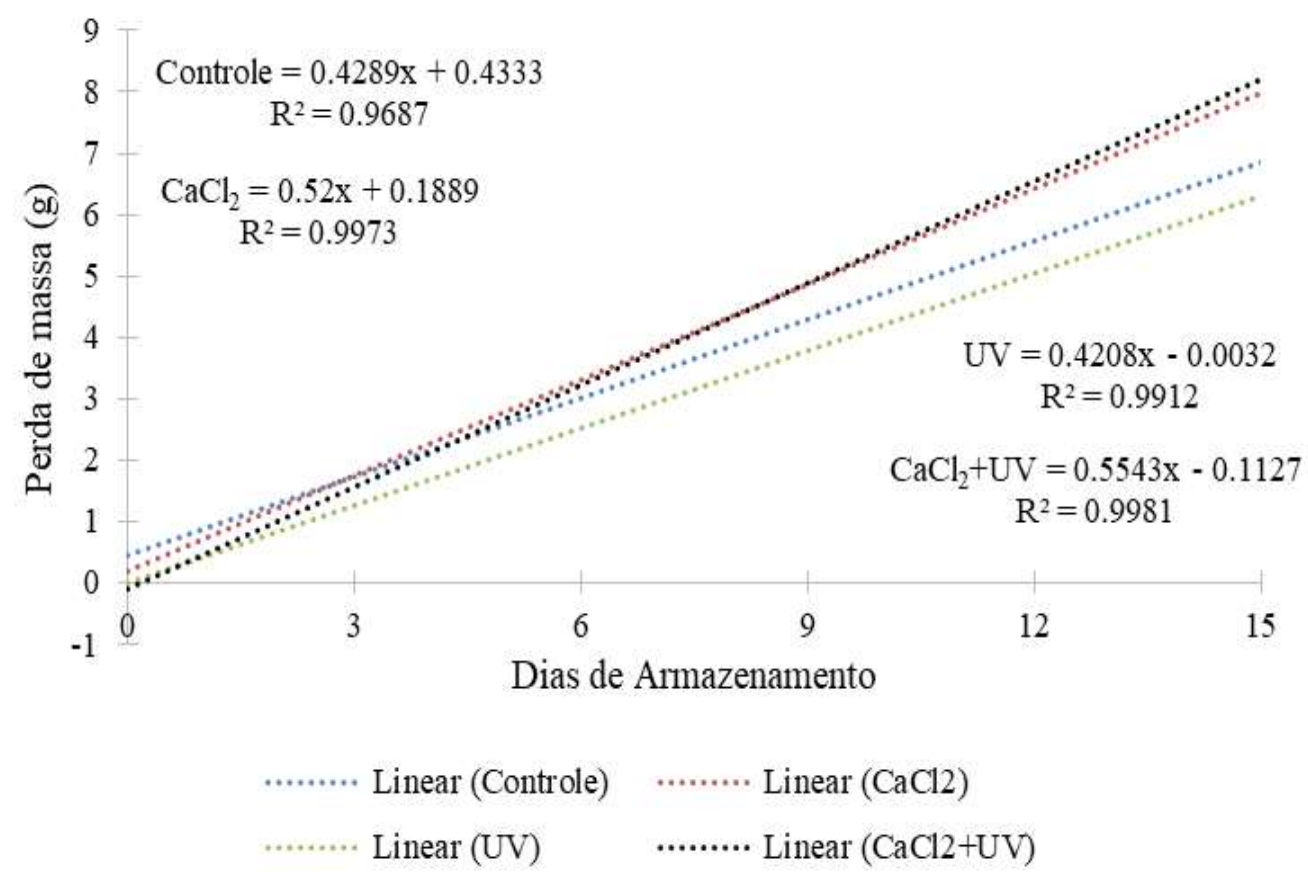

Fonte: Autores.

Figura 2: Firmeza de frutos de tomate submetidos à imersão em Cloreto de Cálcio e exposição à radiação UV.

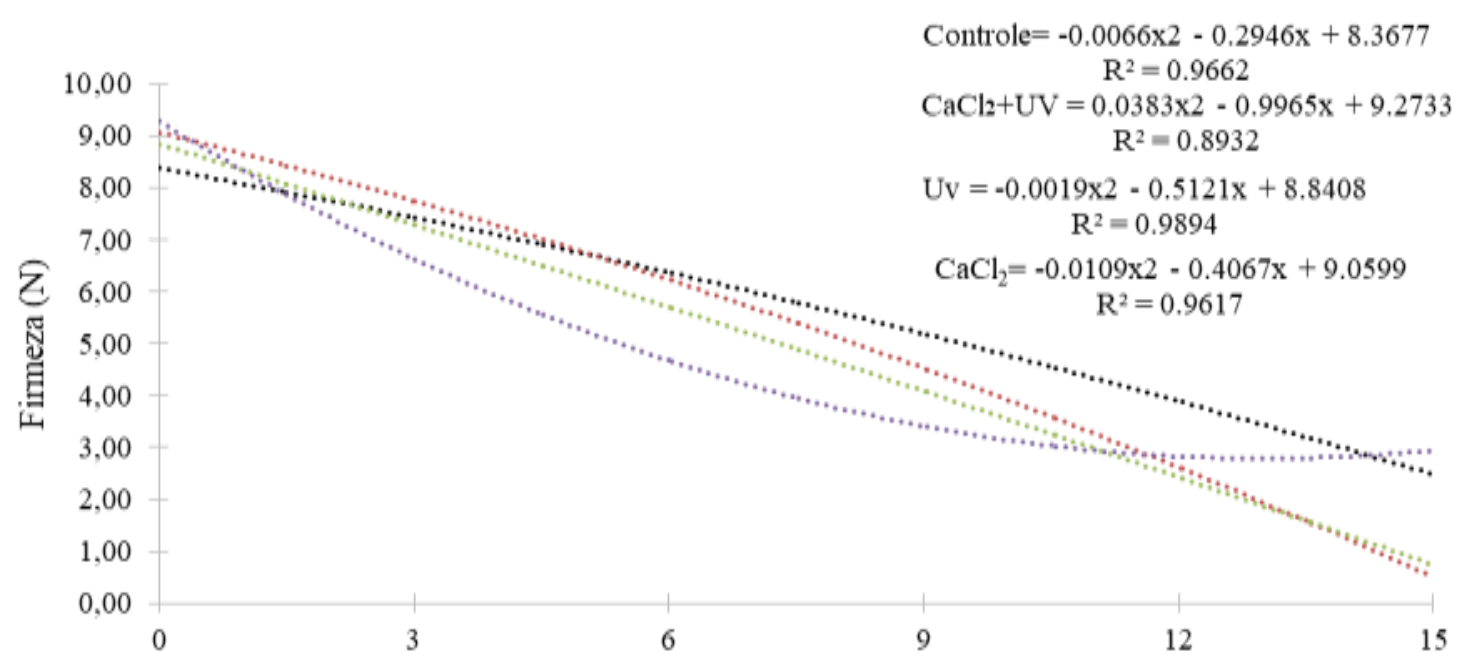

Dias de Armazenamento

Polinômio (Controle)

Polinômio (UV)
Polinômio $(\mathrm{CaCl} 2)$

Polinômio $(\mathrm{CaCl} 2+\mathrm{UV})$

Fonte: Autores.

Com relação à variação de coloração dos frutos, verificou-se que a variável *L para os frutos de tomate diferiu $(\mathrm{p}<0,05)$ com a imersão em $\mathrm{CaCl}_{2}$ e exposição UV ao longo do período de armazenamento (Figura 3). Maior *L foi obtido para os frutos expostos à luz UV (50,9) (Figura 3). Os frutos submetidos aos tratamentos $\mathrm{Controle} \mathrm{CaCl}_{2} \mathrm{e} \mathrm{CaCl}+\mathrm{UV}$ obtiveram menores valores de $* \mathrm{~L}$ iguais a 47,0, 48,3 e 48,8 respectivamente (Figura 3A). Ao longo do período de 
armazenamento, no período zero o valor de *L foi superior aos demais dias de avaliação $(58,1)$. Valores intermediários de *L foram obtidos nos dias de avaliação $3(54,2)$ e $6(49,1)$. Menores valores desta variável foram obtidos nos dias de avaliação 9 $(44,1), 12(44,5)$ e $15(41,9)$ (Figura 4B).

Figura 3: *L de tomate submetidos à imersão em Cloreto de Cálcio e exposição à radiação UV (A) e durante o armazenamento (B).
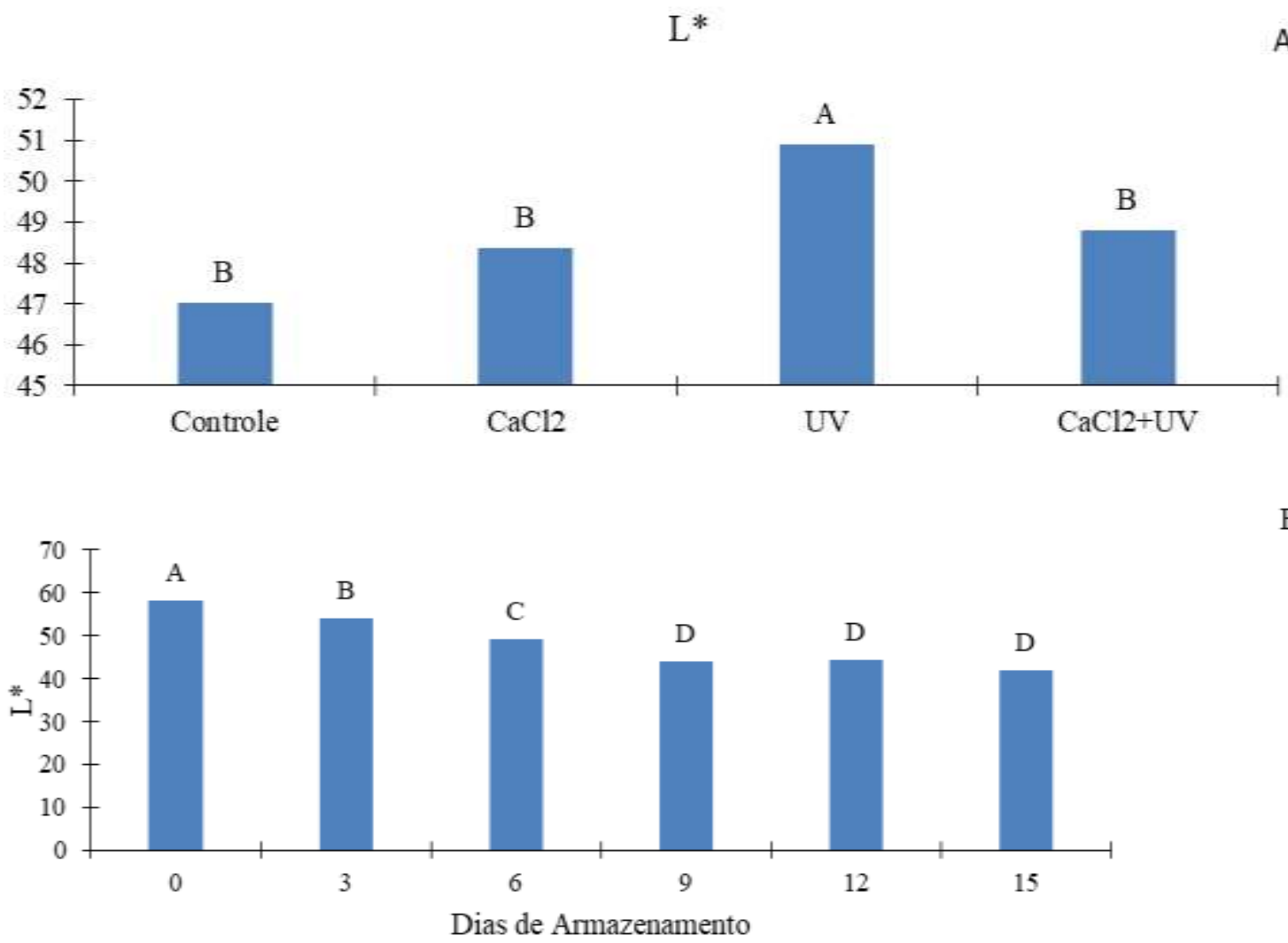

*Médias seguidas de letras iguais não diferem estatisticamente pelo teste de Tukey a 5\% de probabilidade. Fonte: Autores.

Frutos submetidos ao tratamento com UV apresentaram maior luminosidade, comparativamente aos demais tratamentos (Figura 3A). Foi possível verificar também a perda da luminosidade dos frutos de tomate, durante o período de armazenamento (Figura 3B). Resultados semelhantes foram observados por Severo et al., (2015) neste estudo frutos de tomate foram embalados e tratados com radiação de UV-C, os autores relatam que embora a radiação UV-C estimulou a produção de etileno, a evolução do amadurecimento dos frutos foi retardada. O uso de UV pode retardar alguns processos fisiológicos dos frutos, como a degradação de clorofilas, mantendo os frutos verdes por um maior período de tempo, e a produção de etileno influenciando desta forma no processo de senescência (Sanches et al., 2017). O etileno é um hormônio vegetal natural produzido por muitos frutos, é responsável pelo aumento da taxa de respiração, e desta forma, acelera o processo de senescência, altas concentrações de etileno no ar durante a pós-colheita provoca amadurecimento, amaciamento da polpa, aumento das doenças de armazenamento e mudanças na coloração de frutos. (Mansourbahmani et al., 2017). Isto significa que com o passar do tempo de armazenamento ocorrerá à diminuição dos pigmentos verdes em estímulo do etileno, consequentemente acontecerá um maior estímulo para degradação da clorofila acelerando a perda da coloração verde, o que resultará na aparência de um produto maduro (Camilo, 2009). 
Para a variável *a houve efeito de interação $(\mathrm{p}<0,05)$ entre os tratamentos com imersão em $\mathrm{CaCl}_{2}$ e exposição UV e os dias de armazenamento (Figura 4).

Durante o período de armazenamento 0 os valores de *a não diferiram entre os tratamentos. No $3^{\circ}$ dia de avaliação os frutos submetidos à exposição à radiação UV e ao tratamento com CaCl2+UV apresentaram maiores médias de *a. No $6^{\circ}$ e $9^{\circ}$ os frutos submersos em $\mathrm{CaCl} 2$ apresentaram maior valor de * a em relação ao tratamento controle, porém não diferiu estatisticamente do tratamento UV e CaCl2+UV. Para o $12^{\circ}$ dia de avaliação verificou-se maiores valores de *a para os tratamentos controle e $\mathrm{CaCl} 2$, e menor valor para o tratamento apenas com UV. No $15^{\circ}$ dia de avaliação, os frutos submetidos à imersão em $\mathrm{CaCl} 2$ obtiveram maior valor de *a e o menor valor foi obtido para o tratamento CaCl2+UV (Figura 4).

Figura 4: *a de frutos de tomate submetidos à imersão em Cloreto de Cálcio e exposição à radiação UV.

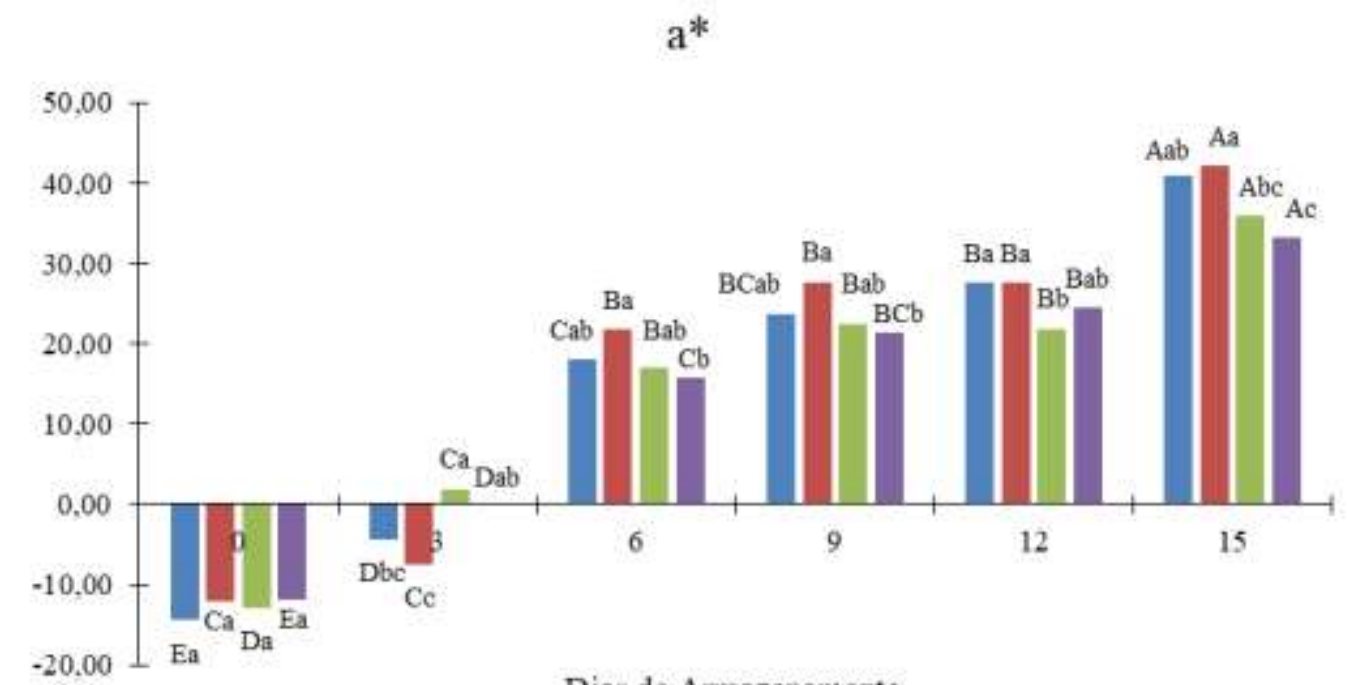

Dias de Armazenamento

acontrole $=\mathrm{CaCl} 2$ = $\mathrm{UV}=\mathrm{CaCl} 2+\mathrm{UV}$

*Médias seguidas de letras iguais não diferem estatisticamente pelo teste de Tukey a 5\% de probabilidade. Fonte: Autores.

A degradação das clorofilas, característico da perda da coloração verde, durante o processo de amadurecimento dos frutos faz parte de uma etapa natural do desenvolvimento. Essa degradação pode ser dada por inúmeros fatores, tais como $\mathrm{pH}$, atividade de enzimas como a clorofilase, temperaturas, luz e oxigênio (Bohn \& Walczyk, 2004).

A variável *b para os frutos de tomate diferiu $(\mathrm{p}<0,05)$ com a imersão em $\mathrm{CaCl}_{2}$ e exposição UV ao longo do período de armazenamento. Os frutos submetidos ao tratamento com exposição ao UV apresentaram valor intermediário de *b $(28,8)$. Os frutos submetidos à imersão em $\mathrm{CaCl}_{2}$ e exposição ao UV apresentaram menor valor de *b (26,5) (Figura 5A). Ao longo do período de armazenamento, $1^{\circ} \mathrm{o}$ valor de *b foi superior aos demais dias de avaliação $(41,8)$. Valores intermediários de *b foram obtidos nos dias de avaliação $0(27,5), 3(30,4), 6(30,1)$ e $12(27,7)$ Menor valor desta variável foi obtido nos dias de avaliação 9 (27,2) (Figura 5B). 
Figura 5: *b de tomate submetidos à imersão em Cloreto de Cálcio e exposição à radiação UV (A) e durante o armazenamento (B).
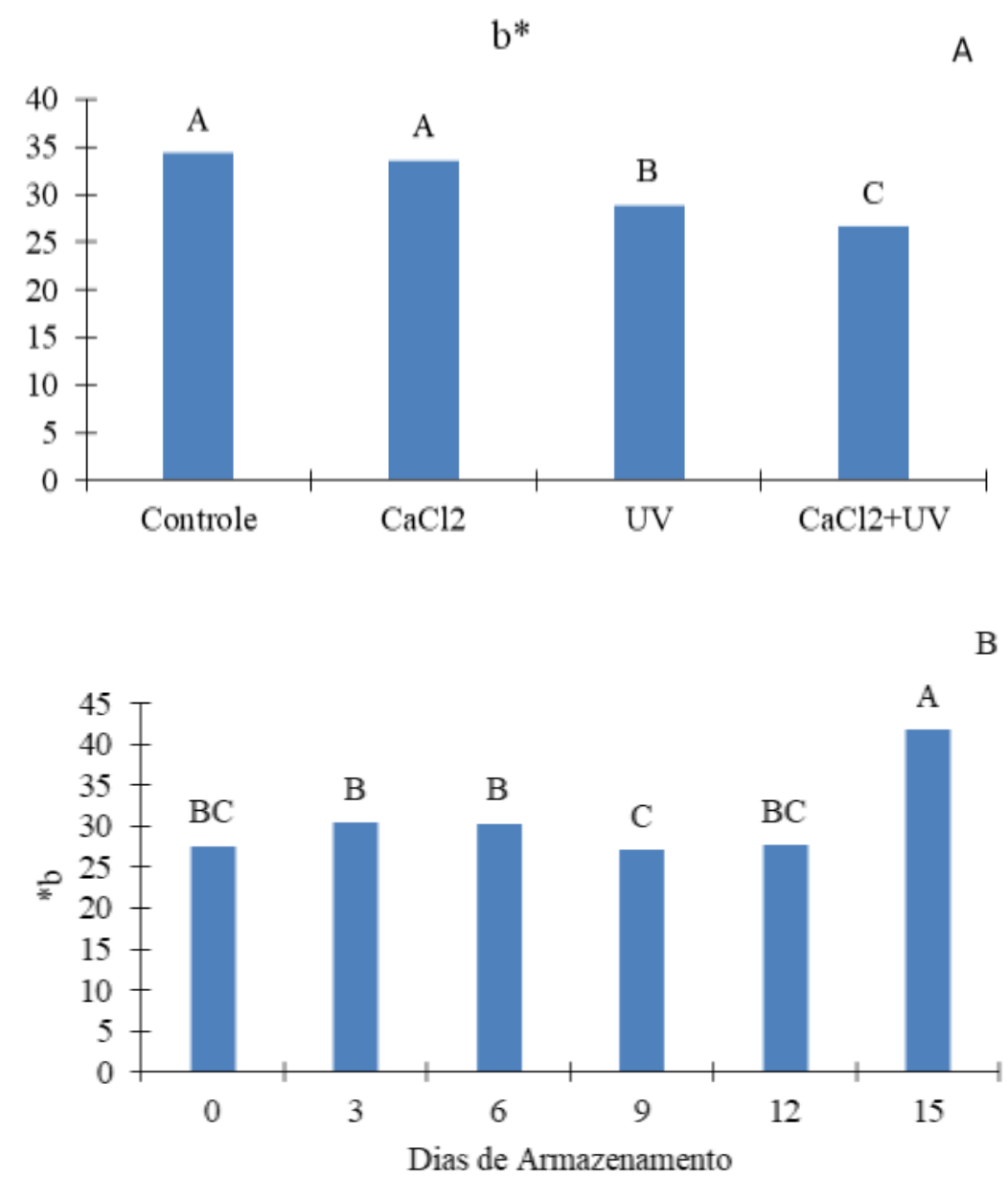

*Médias seguidas de letras iguais não diferem estatisticamente pelo teste de Tukey a 5\% de probabilidade. Fonte: Autores.

Para a maioria dos frutos o amadurecimento envolve alterações da coloração (Oliveira et al., 2021). Em geral, estas alterações ocorrem com a perda da coloração verde, característica do fruto imaturo, para outra cor, vermelha no caso do tomate. Esta mudança está relacionada ao conteúdo de pigmentos totais, como clorofilas $a, b$ e carotenoides, sendo estes pigmentos degradados ou sintetizados durante o processo de amadurecimento (Bohn \& Walczyk, 2004).

Após a colheita, os frutos passam a utilizar suas próprias reservas para continuar o seu desenvolvimento. Porém, a energia liberada pela respiração pode ser utilizada, em alguns casos, para continuar a síntese de pigmentos, enzimas e outros materiais de estrutura molecular elaborada (Chitarra \& Chitarra, 2005).

O teor de Sólidos Solúveis Totais variou ao longo do período de armazenamento nos frutos submetidos aos diferentes tratamentos (Tabela. 1). Até o terceiro dia de avaliação verificou-se aumento de SST para os frutos do tratamento controle e do tratamento com $\mathrm{CaCl}_{2}$. No entanto, a partir do sexto dia de avaliação os frutos do tratamento controle passaram a aumentar o teor de SST, enquanto para os demais tratamentos o aumento se deu no $12^{\circ}$ dia de avaliação. Menezes et al., (2017) analisando frutos de tomates tratados com cloreto de cálcio observou que os teores de sólidos solúveis totais (SST) apresentaram uma tendência de aumento até o nono dia de avaliação dos frutos tratados com cloreto de cálcio $\left(6,18{ }^{\circ}\right.$ Brix $)$ com posterior redução no último dia de avaliação. No caso de frutos, os sólidos solúveis totais têm tendência a exibir maior concentração com a evolução da maturação, devido aos processos de biossíntese. Os teores de SST encontrados nos tomates podem estar 
relacionados ao grau de amadurecimento, pois amostras constituídas de frutos mais maduros apresentaram maior teor de SST. (Kluge \& Minami, 1997). Baseado nos valores encontrados observa-se a influência da variável tempo e dos tratamentos nos teores de sólidos solúveis dos frutos, uma vez que houve a oscilação desta variável durante o experimento. Além do período de armazenamento, existem outros fatores que influenciam nos teores de SST, como por exemplo, o efeito da temperatura, descrito por Miranda et al. (2002) que ao analisarem o armazenamento de dois tipos de sapoti em temperatura ambiente, observaram que não houve diferenças significativas entre as atmosferas utilizadas, nem entre os tipos de frutos em relação ao teor médio de sólidos solúveis totais (SST).

Entretanto, Neres et al., (2004) avaliando o efeito da temperatura de armazenamento e do uso de embalagem de polietileno de baixa densidade na conservação pós-colheita de jiló, verificaram que o teor de SST aumentou no início do período de armazenamento, e que os frutos armazenados a $25^{\circ} \mathrm{C}$ apresentaram menores teores de SST. Os autores atribuíram esse comportamento ao avanço do processo de maturação demonstrado pela evolução da cor. Esse comportamento pode explicar as alterações de cor obtidas no presente trabalho. No entanto, diversos fatores, tais como, genéticos, estádios de maturação do fruto, processos transpiratórios do fruto, dentre outros tem um papel importante no teor de SST.

Foi possível verificar o aumento do $\mathrm{pH}$ em todos os frutos submetidos aos diferentes tratamentos. Porém no $3^{\circ} \mathrm{e} 9^{\circ}$ dia verificou-se que frutos tratados com $\mathrm{UV}$ apresentaram menores valores de $\mathrm{pH}$, mas no $15^{\circ}$ dia esses valores de $\mathrm{pH}$ foram muito próximos para todos os tratamentos (Tabela.1). Com o aumento do $\mathrm{pH}$, a acidez titulável foi drasticamente reduzida ao longo do tempo para todos os tratamentos (Tabela.1). Lima et al., (2018), observaram em jabuticaba, que a acidez diminuía com o avanço do estádio de maturação. França (2017), em estudos sobre as características físico-químicas da cebola, observou que a redução da acidez ocorre normalmente em hortaliças e faz parte do processo de senescência, sendo ocasionada pela possível perda de ácidos orgânicos em virtude da drenagem do líquido celular e volatilização dos ácidos presentes.

Durante o processo de armazenamento é comum observar um incremento dos teores se SST, aumento dos valores de $\mathrm{pH}$, redução da acidez titulável e maiores valores da relação SS/AT de frutos de tomate. Isto ocorre porque no processo de amadurecimento dos frutos há uma diminuição nos teores dos ácidos orgânicos, a redução da acidez é também resultante do stress causado pela colheita e durante a armazenagem, devido à oxidação desses compostos na produção de energia via ciclo de Krebs para manter os processos vitais (Barankevicz et al., 2015). 
Research, Society and Development, v. 10, n. 14, e218101421887, 2021

(CC BY 4.0) | ISSN 2525-3409 | DOI: http://dx.doi.org/10.33448/rsd-v10i14.21887

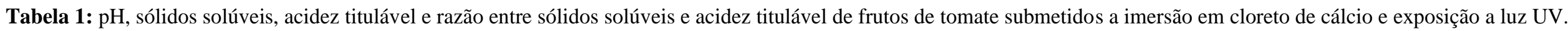

\begin{tabular}{|c|c|c|c|c|c|c|c|}
\hline \multirow{3}{*}{ Tratamentos } & \multicolumn{5}{|c|}{ Dias de armazenamento } & \multirow[b]{2}{*}{15} & \multirow{3}{*}{$\begin{array}{c}\mathrm{CV} \\
\%\end{array}$} \\
\hline & 0 & 3 & 6 & 9 & 12 & & \\
\hline & \multicolumn{6}{|c|}{$\mathrm{pH}$} & \\
\hline Controle & $3,67 \mathrm{Ea}$ & $3,96 \mathrm{Dbc}$ & $4,05 \mathrm{Cab}$ & $4,03 \mathrm{BCab}$ & $4,43 \mathrm{Ba}$ & $4,46 \mathrm{Aab}$ & \multirow{4}{*}{1,9} \\
\hline $\mathrm{CaCl} 2$ & $3,67 \mathrm{Ca}$ & $4,07 \mathrm{Cc}$ & $4,17 \mathrm{Ba}$ & $4,15 \mathrm{Ba}$ & $4,46 \mathrm{Ba}$ & $4,22 \mathrm{Aa}$ & \\
\hline UV & $3,67 \mathrm{Da}$ & $3,74 \mathrm{Ca}$ & $4,22 \mathrm{Bab}$ & $3,82 \mathrm{Bb}$ & $4,36 \mathrm{Bb}$ & $4,32 \mathrm{Abc}$ & \\
\hline $\mathrm{CaCl} 2+\mathrm{UV}$ & $3,67 \mathrm{Ea}$ & $3,88 \mathrm{Dab}$ & $4,17 \mathrm{Cb}$ & 4,07BCab & $4,40 \mathrm{Bab}$ & $4,40 \mathrm{Ac}$ & \\
\hline \multicolumn{8}{|c|}{ Sólidos Solúveis $\left({ }^{\circ}\right.$ Brix $)$} \\
\hline Controle & $3,97 \mathrm{BCa}$ & $4,65 \mathrm{Aa}$ & $3,60 \mathrm{Ca}$ & $4,05 \mathrm{ABCab}$ & $4,25 \mathrm{Aba}$ & $4,35 \mathrm{Aba}$ & \multirow{4}{*}{6,6} \\
\hline $\mathrm{CaCl} 2$ & 3,97Aba & 4,40Aab & $4,05 \mathrm{ABa}$ & $4,10 \mathrm{ABa}$ & $3,55 \mathrm{Cb}$ & 4,20Aa & \\
\hline UV & 3,97Aa & $3,60 \mathrm{Ac}$ & $3,75 \mathrm{Aa}$ & 3,95Aab & $3,65 \mathrm{Ab}$ & $4,15 \mathrm{Aa}$ & \\
\hline $\mathrm{CaCl} 2+\mathrm{UV}$ & 3,97Aba & $3,90 \mathrm{ABbc}$ & $3,95 \mathrm{ABa}$ & $3,50 \mathrm{ABb}$ & $3,35 \mathrm{Cb}$ & 4,00Aa & \\
\hline \multicolumn{8}{|c|}{ Acidez Titulável } \\
\hline Controle & $6,95 \mathrm{Aa}$ & $4,85 \mathrm{BCb}$ & $4,45 \mathrm{CDa}$ & $5,15 \mathrm{Bb}$ & $4,05 \mathrm{Da}$ & $4,37 \mathrm{CDb}$ & \multirow{4}{*}{5,12} \\
\hline $\mathrm{CaCl} 2$ & $6,95 \mathrm{Aa}$ & $5,10 \mathrm{Bab}$ & $4,40 \mathrm{Ca}$ & $4,30 \mathrm{Cc}$ & $4,10 \mathrm{CDa}$ & $3,65 \mathrm{Da}$ & \\
\hline UV & $6,95 \mathrm{Aa}$ & $5,45 \mathrm{Ba}$ & $4,25 \mathrm{Ca}$ & $6,75 \mathrm{Aa}$ & $4,45 \mathrm{Ca}$ & $3,90 \mathrm{Cab}$ & \\
\hline $\mathrm{CaCl} 2+\mathrm{UV}$ & $6,95 \mathrm{Aa}$ & $4,69 \mathrm{Bb}$ & $4,05 \mathrm{CDa}$ & $4,60 \mathrm{BCc}$ & $3,90 \mathrm{Da}$ & 4,20BCDa & \\
\hline \multicolumn{8}{|c|}{ Sólidos Solúveis/Acidez Titulável } \\
\hline Controle & $0,57 \mathrm{Ca}$ & $0,96 \mathrm{ABa}$ & $0,81 \mathrm{Bb}$ & $0,79 \mathrm{Bb}$ & $1,05 \mathrm{Aa}$ & $1,00 \mathrm{Ab}$ & \multirow{4}{*}{8,67} \\
\hline $\mathrm{CaCl} 2$ & $0,57 \mathrm{Ca}$ & $0,86 \mathrm{Ba}$ & $0,92 \mathrm{Bab}$ & $0,96 \mathrm{Ba}$ & $0,87 \mathrm{Bb}$ & $1,15 \mathrm{Aa}$ & \\
\hline UV & $0,57 \mathrm{Da}$ & $0,66 \mathrm{CDb}$ & $0,88 \mathrm{Bab}$ & $0,59 \mathrm{Dc}$ & $0,82 \mathrm{BCb}$ & $1,07 \mathrm{Aab}$ & \\
\hline $\mathrm{CaCl} 2+\mathrm{UV}$ & $0,57 \mathrm{Ca}$ & $0,83 \mathrm{ABa}$ & $0,98 \mathrm{Aa}$ & $0,77 \mathrm{Bb}$ & $0,86 \mathrm{ABb}$ & $0,95 \mathrm{Ab}$ & \\
\hline
\end{tabular}

*Médias seguidas de mesma letra maiúscula na linha e minúscula na coluna não diferem entre si pelo teste de Tukey. Fonte: Autores. 


\section{Conclusão}

Frutos submetidos à radiação ultravioleta apresentaram menor perda de massa fresca quando comparado aos demais tratamentos.

O uso do cloreto de cálcio junto à radiação UV auxilia na manutenção da firmeza dos frutos ao longo do armazenamento.

Os tratamentos com $\mathrm{CaCl}_{2}$, $\mathrm{UV}$ e $\mathrm{CaCl}_{2}+\mathrm{UV}$ não interferiram nas propriedades químicas dos frutos de tomate durante o processo de pós-colheita, exceto na relação SST/ATT que no $15^{\circ}$ de análises apresentaram os maiores valores. Dessa forma, recomenda-se o uso da radiação UV para retardar o avanço do processo de maturação em tomates. Para trabalhos futuros sugere-se a utilização de outros tempos de exposição a luz UV de forma a aumentar a eficiência dos tratamentos, bem como, a utilização de luz pulsada para aumentar a conservação pós colheita de tomates.

\section{Referências}

Almeida, R. R., Caetano, L. R., Almeida, V. F. R. \& Nascimento, L. M. (2019). Efeito da radiação ultravioleta C na qualidade e armazenamento pós-colheita de pequi. Nativa, Sinop, 7(1): 29-36

Arenas, L. A. O. (2014). Desenvolvimento de estrutura para purificação de água potável, através da Irradiação de UV com lâmpadas fluorescentes especiais. (Dissertação de mestrado em Engenharia Elétrica). Universidade Estadual Paulista Júlio de Mesquita Filho, Faculdade de Engenharia.

Barankevicz, G. B., Novello, D., Resende, J. T., Schwarz, K., \& Santos, E. F. (2015). Características físicas e químicas da polpa de híbridos de tomateiro, durante o armazenamento congelado. Horticultura Brasileira, 33, 7-11.

Bohn, T., \& Walczyk, T. (2004). Determination of chlorophyll in plant samples by liquid chromatography using zinc-phthalocyanine as an internal standard. Journal of chromatography A, 1024(1-2), 123-8.

Bragança, T. G. (2021) Efeito da atmosfera modificada na conservação pós-colheita de frutos do maracujazeiro-amarelo (Passiflora edulis F. Flavicarpa). Brazilian Journal of Development, Curitiba, 7(8): 82181-82198.

Camillo, M. F. (2009). Danos mecânicos e seus efeitos na qualidade pós-colheita de frutos de caroço.

Campos, A. J. D., Vieites, R. L., Neves, L. C., Moura, M. L. D. S., Moura, E. A. D., \& Correio, K. G. M. D. A. (2015). Qualidade do tomate'Pitenza'com utilização da radiação ultravioleta (UV-C). Horticultura Brasileira, 300-7.

Carnelossi, M. A. G., Santos, P. T. M., Constant, P. B. L., Matos, G. B., Moreira, M. N., Costa, R. I. S. A., Soares, A. C., Santos, L. de S. Reis, A. A., Matos, P. N. \& Gagliardi, P. R. (2021) Uso de revestimento de fécula de mandioca e lactato de cálcio em mamão minimamente processado. Research, Society and Development, [S. l.], 10(11): e373101119508, 2021. 10.33448/rsd-v10i11.19508. https://rsdjournal.org/index.php/rsd/article/view/19508. Acesso em: 14 out. 2021.

Chien, P. J., Lin, H. R., \& Su, M. S. (2013). Effects of edible micronized chitosan coating on quality and shelf life of sliced papaya. Food and Nutrition, 4, p. $9-13$.

Chitarra, M. I. F., \& Chitarra, A. B. (1990). Pós-colheita de frutos e hortaliças: fisiologia e manuseio (p. 320). Esal/Faepe.

Chitarra, M. I. F., \& Chitarra, A. B. (2005). Pós-Colheita de Frutas e Hortaliças: Fisiologia e Manuseio. (2a ed.).

Estrela, C. (2018). Metodologia Científica: Ciência, Ensino, Pesquisa. Editora Artes Médicas.

Ferraz, E., Evangelista, R., Cláudio, M., Soares, L., Silva, B., \& Cardoso, A. (2012). Características físico-químicas em tomates cereja tipo Sweet Grape envolvidos por diferentes películas protetoras. Horticultura Brasileira, Brasília, 30(2): 7115-22.

França, F. D. D. (2017). Conservação pós-colheita de cebola em função da adubação nitrogenada.

IBGE. (2019). Levantamento sistemático da produção agrícola-janeiro 2021. https:// biblioteca.ibge.gov.br/visualizacao/periodicos/2415/e pag_2021_jan.pdf

Khaliq, G., Mohamed, M. T. M., Ali, A., Ding, P., \& Ghazali, H. M. (2015). Effect of gum arabic coating combined with calcium chloride on physicochemical and qualitative properties of mango (Mangifera indica L.) fruit during low temperature storage. Scientia Horticulturae, $190,187-94$.

Kluge, R. A., \& Minami, K. (1997). Efeito de ésteres de sacarose no armazenamento de tomatesSanta Clara'. Scientia Agrícola, 54(1-2): 39-44.

Lima, T. L. B; carneiro, E. F. Sá; silva, R. M; Figueirêdo, R. M. F; \& Queiroz, A. J. M. (2018). Caracterização físico-química da casca de jabuticaba em diferentes estádios fisiológicos. Congresso Técnico Científico da Engenharia e da Agronomia.

Linhares, L. A., Dos Santos, C. D., De Abreu, C. M. P. \& Corrêa, A. D. (2007). Transformações Químicas, Físicas E Enzimáticasde Goiabas 'Pedro Sato' Tratadas Na Pós-Colheita Com Cloreto De Cálcio E 1-Metilciclopropeno E Armazenadas Sob Refrigeração. Ciência E Tecnologia De Alimentos. 31 (3).

Mansourbahmani, S., Ghareyazie, B., Kalatejari, S., Mohammadi, R. S., \& Zarinnia, V. (2017). Effect of post-harvest UV-C irradiation and calcium chloride on enzymatic activity and decay of tomato (Lycopersicon esculentum L.) fruit during storage. Journal of integrative agriculture, 16(9): 2093-100. 
Menezes, K. R. P., de Souza Santos, G. C., de Oliveira, O. M., Sanches, A. G., Cordeiro, C. A. M., \& de Oliveira, A. R. G. (2017). Influência Do Revestimento Comestível Na Preservação Da Qualidade Pós-Colheita De Tomate De Mesa. In Colloquium Agrariae. 13(3), 14-28.

Miranda, M. R. A. D., Silva, F. S. D., Alves, R. E., Filgueiras, H. A. C., \& Araújo, N. C. C. (2002). Armazenamento de dois tipos de sapoti sob condição de ambiente. Revista Brasileira de Fruticultura, 24, 644-6.

Moraes, I. P., Leite, A. R. Z., Filho, S. M. P., Bortoluzzi, L. C. P., Marques, L. L. M., Leimann, F. V., Poliseli-Scopel, F. H. \& Perdoncini, M. R. F. G. (2021). Tratamentos de morangos por radiação UV -C emitidos por lâmpadas de LED. Brazilian Journal of Development, Curitiba, 7(4); $37119-37128$.

Neres, C. R. L., Vieira, G., Diniz, E. R., Mota, W. F. D., \& Puiatti, M. (2004). Conservação do jiló em função da temperatura de armazenamento e do filme de polietileno de baixa densidade. Bragantia, 63, 431-8.

Neto, J. S., Schwan-estrada, K. R. F., Sena, J. O. A., Jardinetti, V. A., Alencar, M. S. R. (2016). Qualidade de frutos de tomateiro cultivado em sistema de produção orgânico e tratados com subprodutos de capim limão. Ciência Agronômica, Fortaleza, 47(4): 633-642.

Oliveira, C. D., Gomes, B. A. F., Furtado, M. L. B., Barbosa, D. P. \& Boas, E. V. de B. V. Alterações nas características físicas e bioquímicas de frutos de Cordia superba durante a maturação. Research, Society and Development, [S. l.], 10(10): e503101019203, $2021.10 .33448 /$ rsd-v10i10.19203. https://rsdjournal.org/index.php/rsd/article/view/19203.

Paixão, A. R. D. C. (2016). Ação da pectina metil esterase e cloreto de cálcio no armazenamento e controle da podridão-mole em pimentão.

Rashida, E.El Fadil, E. B., \& El Tinay, A. H. (1997). Changes in chemical composition of guava fruits during development and ripening. Food Chemistry, 59(3), 395-9.

Sanches, A. G., Costa, J. M., Silva, M. B. da., Moreira, E. G. S., Santana, P. J. A. \& Cordeiro, C. A. M. (2017). Aspectos qualitativos e amadurecimento do araçá amarelo tratado com radiação UV-C. Nativa, Sinop, 5(5): 303-310.

Severo, J., Tiecher, A., Pirrello, J., Regad, F., Latché, A., Pech, J. C., Bouzayen, M. \& Cesar ValmorRombaldi, C. V. (2015). UVC radiation modifies the ripening and accumulation of ethylene response factor (ERF) transcripts in tomato fruit. Postharvest Biology and Technology, $102,9-16$.

Song, L., Wang, Z., Wang, Z., Meng, G., Zhai, R., Cai, M., \& Xu, L. (2016). Screening of cell wall-related genes that are expressed differentially during ripening of pears with different softening characteristics. Postharvest Biology and Technology, 115, 1-8.

Vasconcelos, L. H. C., Silva, F. A. da., Nascimento, L. M. \& Vasconcelos, R. F. (2020). Post-harvest evaluation of 'Dekopon' tangerins submitted to the application of calcium chloride in pre-harvest. Research, Society and Development, [S. l.], 9(6): e132963638.

Yamamoto, E. L. M., de Araújo Ferreira, R. M., de Oliveira Fernandes, P. L., Albuquerque, L. B., \& de Oliveira Alves, E. (2011). Função do cálcio na degradação da parede celular vegetal de frutos. Revista verde de agroecologia e desenvolvimento sustentável, $6(2), 6$. 